Copyright by the AIP Publishing. Park, Chee-Sung; Khachaturyan, Armen; Priya, Shashank, "Giant magnetoelectric coupling in laminate thin film structure grown on magnetostrictive substrate," Appl. Phys. Lett. 100, 192904 (2012); http:// dx.doi.org/10.1063/1.4712132

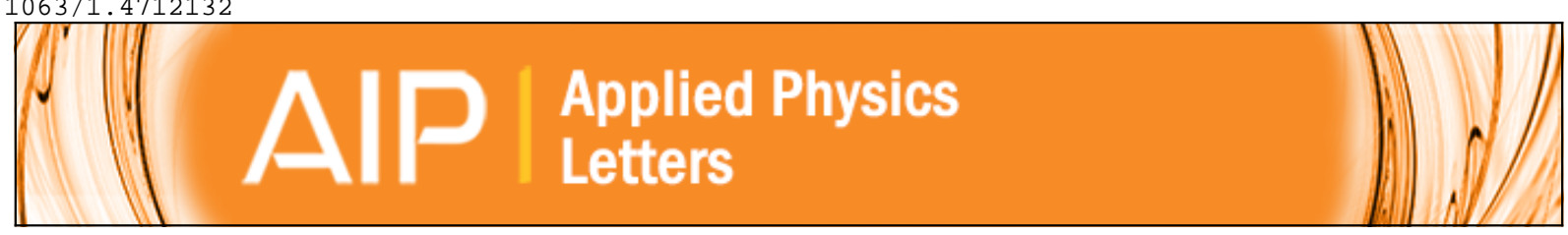

\title{
Giant magnetoelectric coupling in laminate thin film structure grown on magnetostrictive substrate
}

Chee-Sung Park, Armen Khachaturyan, and Shashank Priya

Citation: Applied Physics Letters 100, 192904 (2012); doi: 10.1063/1.4712132

View online: http://dx.doi.org/10.1063/1.4712132

View Table of Contents: http://scitation.aip.org/content/aip/journal/apl/100/19?ver=pdfcov

Published by the AIP Publishing

\section{Articles you may be interested in}

Giant self-biased magnetoelectric coupling in co-fired textured layered composites

Appl. Phys. Lett. 102, 052907 (2013); 10.1063/1.4791685

Modeling of resonant magneto-electric effect in a magnetostrictive and piezoelectric laminate composite structure coupled by a bonding material

J. Appl. Phys. 112, 064109 (2012); 10.1063/1.4752271

Dual-resonance converse magnetoelectric and voltage step-up effects in laminated composite of long-type $0.71 \mathrm{~Pb}(\mathrm{Mg} 1 / 3 \mathrm{Nb} 2 / 3) \mathrm{O} 3-0.29 \mathrm{PbTiO} 3$ piezoelectric single-crystal transformer and Tb0.3Dy0.7Fe1.92 magnetostrictive alloy bars

J. Appl. Phys. 109, 104103 (2011); 10.1063/1.3587574

Strong magnetoelectric charge coupling in stress-biased multilayer-piezoelectricmagnetostrictive composites J. Appl. Phys. 101, 124102 (2007); 10.1063/1.2748712

Push-pull mode magnetostrictive/piezoelectric laminate composite with an enhanced magnetoelectric voltage coefficient

Appl. Phys. Lett. 87, 062502 (2005); 10.1063/1.2007868

\section{$\underset{\substack{\text { Aubloning } \\ \text { ald }}}{A}$ Re-register for Table of Content Alerts}




\title{
Giant magnetoelectric coupling in laminate thin film structure grown on magnetostrictive substrate
}

\author{
Chee-Sung Park, ${ }^{1}$ Armen Khachaturyan, ${ }^{2}$ and Shashank Priya ${ }^{1, a)}$ \\ ${ }^{1}$ Center for Energy Harvesting Materials and Systems (CEHMS), Virginia Polytechnic Institute and State \\ University, Blacksburg, Virginia 24061, USA \\ ${ }^{2}$ Rutgers, The State University of New Jersey, Piscataway, New Jersey 08854, USA
}

(Received 25 March 2012; accepted 21 April 2012; published online 8 May 2012)

\begin{abstract}
Highly dense $1 \mu \mathrm{m}$-thick piezoelectric film was deposited on magnetostrictive substrate [platinized nickel-zinc ferrite (NZF)]. A strong magnetic coupling between the piezoelectric film and magnetostrictive NZF substrate was measured exhibiting the maximum magnetoelectric (ME) coefficient on the order of $140 \mathrm{mV} / \mathrm{cm}$ Oe at the conditions of $\mathrm{H}_{\mathrm{DC}}=50 \mathrm{Oe}$ and $\mathrm{H}_{\mathrm{AC}}=1 \mathrm{Oe}$ at $f=1 \mathrm{kHz}$. This giant ME coupling under low DC magnetic field condition is attributed to effective elastic coupling. A rotation-type dynamic strain distribution was observed on the PZT film surface which provides information about the nature of elastic coupling. (c) 2012 American Institute of Physics. [http://dx.doi.org/10.1063/1.4712132]
\end{abstract}

\begin{abstract}
Magnetoelectric (ME) materials are being targeted for applications in filters, phase shifters, electro-magnetic interference (EMI) devices, tunable transformers, and sensor devices. ${ }^{1-4}$ ME effect in composites occurs through the elastic coupling between the piezoelectric and magnetostrictive properties. The role of interface becomes critical in this strain coupling. Recently, much attention has been laid upon nanocomposite films to understand the interfacial dynamics and role of domains. ${ }^{4-8}$ However, there has been no direct measurement on the dynamics of this elastic coupling which is important to understand the observed response. ME coupling in the films has been studied for multiferroic 1-3 vertical $\left[\mathrm{CoFe}_{2} \mathrm{O}_{4}(\mathrm{CFO})-\mathrm{BaTiO}_{3}\right.$ (BTO)] heterostructure film through indirect method (measuring magnetization-magnetic field response and polarization-electric field response to show duality). Direct ME measurement on such nanocomposite structure is difficult because of the low resistance of the ferromagnetic pillars that penetrate through the film. ${ }^{5,6}$ In comparison, ME coupling has been successfully measured in thin films with CFO nanoparticle embedded in $\mathrm{Pb}\left(\mathrm{Zr}_{0.6} \mathrm{Ti}_{0.4}\right) \mathrm{O}_{3}$ (PZT) matrix (3-0 type), ${ }^{7}$ and in CFO/PZT laminate thin film (2-2 type) synthesized by pulsed-laser deposition. ${ }^{8}$ However, most of the ME composite thin films reported in literature based upon the ferrite-perovskite structure show quite low ME values mainly due to the fact that the response is clamped by substrate and difficulty in fully poling the piezoelectric phase. ${ }^{4,9,10}$ It is well-known that the mechanical and electrical properties of films are strongly dependent upon the substrate. Furthermore, if one can separate the piezoelectric film from the magnetic layer of lower resistivity then better poling can be achieved. In this study, we demonstrate growth of highly dense piezoelectric film on a polycrystalline magnetostrictive substrate which reduces substrate clamping effect and maximizes the strain coupling. This is highly cost-effective structure as it utilizes cheap ferrite substrate synthesized through conventional sintering process. In-situ dynamic coupling was monitored by using
\end{abstract}

\footnotetext{
${ }^{\text {a) }}$ Author to whom correspondence should be addressed. Electronic mail: spriya@vt.edu.
}

non-contact laser vibrometry. Based upon the results, we provide explanation for the strain transfer process occurring in perovskite-ferrite film-substrate system.

$\mathrm{Ni}_{0.8} \mathrm{Zn}_{0.2} \mathrm{Fe}_{2} \mathrm{O}_{4}$ [nickel-zinc ferrite (NZF)] plates were synthesized using conventional mixed oxide method. After mixing $\mathrm{NiO}, \mathrm{ZnO}$, and $\mathrm{Fe}_{2} \mathrm{O}_{3}$ and drying, the mixture was calcined at $1000{ }^{\circ} \mathrm{C}$ for $4 \mathrm{~h}$. Calcined powders were ballmilled for $24 \mathrm{~h}$, dried, sieved, pressed using cold isostatic pressure (CIP), and sintered at $1250^{\circ} \mathrm{C}$ for $4 \mathrm{~h}$. The sintered plates were machined to be flat with thickness of $550 \mu \mathrm{m}$. One of the surfaces of NZF plate with dimension of $14.5 \mathrm{~mm} \times 14.5 \mathrm{~mm} \times 550 \mu \mathrm{m}$ was polished using $0.25 \mu \mathrm{m}$ alumina slurry. On this surface, $\sim 100 \mathrm{~nm}$-thick platinum (Pt) was deposited as the bottom electrode. Subsequently, PZT film was deposited by sol-gel method using the process described in detail somewhere else. ${ }^{11}$ The $0.4 \mathrm{M}$ $\mathrm{Pb}_{1.1}\left(\mathrm{Zr}_{0.6} \mathrm{Ti}_{0.4}\right) \mathrm{O}_{3}$ solution was deposited by repeating the spin-coating steps at $3000 \mathrm{rpm}$ for $60 \mathrm{~s}$. Deposited films were pyrolysed at $300^{\circ} \mathrm{C}$ for $5 \mathrm{~min}$ and post-annealing was conducted at $650{ }^{\circ} \mathrm{C}$ for $15 \mathrm{~min}$. For top electrodes, $0.5 \mathrm{~mm}$ diameter Pt dots were deposited on the PZT film. The microstructure and composition of the film were investigated by scanning electron microscopy (SEM). The polarization hysteresis loops were measured by using ferroelectric test system (Radiant, Premier II). The magnetization of the composite was measured by using vibrating sample magnetometer (VSM). For the ME voltage coefficient measurement, an electromagnet was used to apply DC magnetic field $\left(\mathrm{H}_{\mathrm{DC}}\right)$ while the sample was held in the center of the Helmholtz coil under an $\mathrm{AC}$ magnetic field $\left(\mathrm{H}_{\mathrm{AC}}\right)$. ME output voltage was monitored using lock-in amplifier. Magnetostriction for NZF substrates was measured by using the strain gauge and Wheatstone Bridge. For dynamic coupling measurement between the film and substrate, a laser vibrometer (Polytech GmbH., MSA-500) was used.

Figure 1(a) shows the schematic of the ME composite structure consisting of PZT film grown on polycrystalline NZF substrate. Figure 1(b) shows the XRD pattern of PZT film deposited on the Pt/NZF at $650^{\circ} \mathrm{C}$ (top) and the NZF substrate (bottom). Perovskite phase was successfully 
(a)

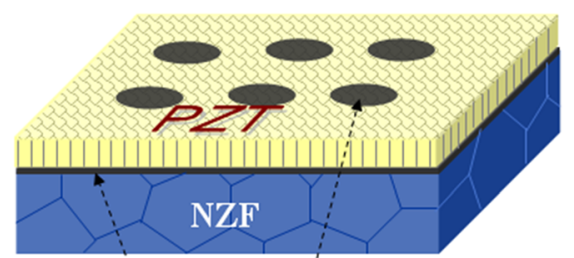

Pt : Bottom and Top Electrodes

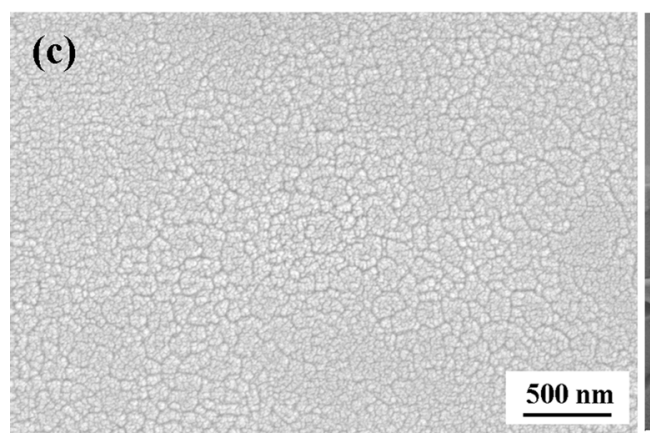

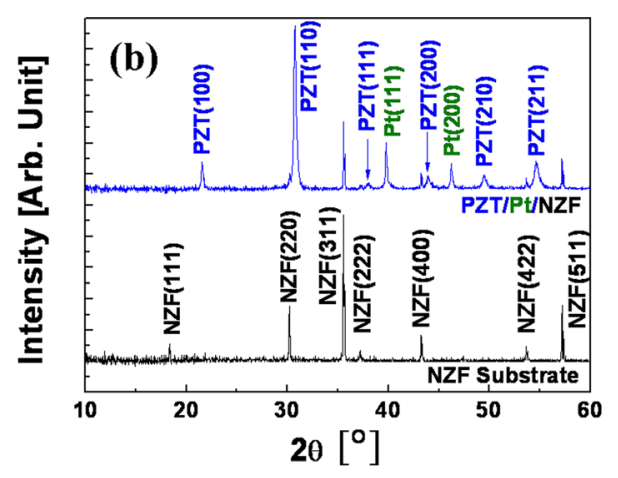

(d)

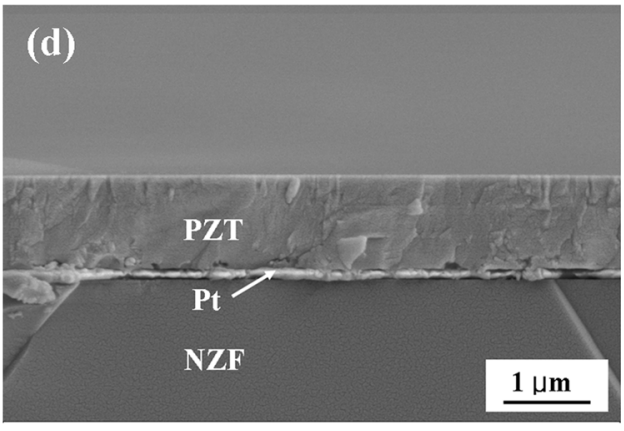

FIG. 1. (a) Schematic diagram of the PZT film deposited on Pt/NZF substrate, (b) XRD patterns of the PZT film on Pt/ NZF substrate: (top) PZT/Pt/NZF and (bottom) NZF. SEM images of the PZT film: (c) plane view and (d) crosssectional view. formed without any trace of secondary phases on the Pt/NZF substrate. Figures 1(c) and 1(d) show the planar and crosssectional views of PZT films. From Fig. 1(c), the in-plane grain size of films was found to be in the range of $\sim 200 \mathrm{~nm}$. From cross-sectional view [Fig. 1(d)], the PZT film was $1 \mu \mathrm{m}$ thick and the Pt layer was $100 \mathrm{~nm}$ thick. The PZT film showed highly dense and homogeneous microstructure without any cracks and delimitations and maintained good adhesion with underlying layers. This indicates that the high quality PZT film was successfully synthesized on the polycrystalline NZF substrate.

Figure 2(a) shows the polarization-electric field (P-E) curves of the PZT films on the Pt/NZF substrate at $f=1 \mathrm{kHz}$. As expected, remanent polarization $\left(\mathrm{P}_{\mathrm{r}}\right)$ and coercive field $\left(E_{c}\right)$ increased with increasing electrical drive. At an applied electric field of $200 \mathrm{kV} / \mathrm{cm}$, the magnitude of $P_{r}$ and $E_{c}$ was measured to be $20.0 \mu \mathrm{C} / \mathrm{cm}^{2}$ and $32.6 \mathrm{kV} / \mathrm{cm}$, respectively. The relative dielectric constant $\left(\mathrm{C} / \mathrm{C}_{\mathrm{o}}\right)$ and loss factor $(\tan \delta)$ of the PZT film under an oscillation voltage of $0.5 \mathrm{~V}_{\mathrm{p}-\mathrm{p}}$ at $f=1 \mathrm{kHz}$ was monitored as a function of DC electric bias as shown in Fig. 2(b). The PZT film shows butterfly-shaped behavior for both dielectric constant and loss which is characteristics of ferroelectric materials and indicates high resistivity. At $f=1 \mathrm{kHz}$ and zero DC field conditions, the dielectric constant and loss values were measured to be 840 and 0.058 , respectively. These results on the PZT film grown on $\mathrm{Pt} / \mathrm{NZF}$ substrate are comparable to a PZT film grown on the $\mathrm{Pt} / \mathrm{Ti} / \mathrm{SiO}_{2} / \mathrm{Si}$ substrate. ${ }^{12,13}$

The magnetization $(\mathrm{M})$ of the PZT/Pt/NZF structure as a function of magnetic field was measured by VSM in longitudinal direction (L-mode) as shown in Fig. 3(a). The coercive magnetic field $\left(\mathrm{H}_{\mathrm{c}}\right)$ and remanent magnetization $\left(\mathrm{M}_{\mathrm{r}}\right)$ were found to be $5 \mathrm{Oe}$ and $6 \mathrm{emu} / \mathrm{cm}^{3}$, respectively and the saturation magnetization was measured to be $315 \mathrm{emu} / \mathrm{cm}^{3}$. This low field saturated magnetization behavior is related to the position of ME peak appearing at low magnetic DC bias. ${ }^{14}$
The ME behavior can be correlated with magnetostriction $(\lambda)$ and magnetization (M) by noticing: ${ }^{15,16}$

$$
\varphi \sim \frac{3 \lambda \sigma}{\left(K+2 \pi M^{2}\right)} \quad \text { or } \quad \lambda \propto M^{2},
$$

where $\varphi$ is the angle of magnetic moments, $K$ and $\sigma$ are the anisotropy constant and stress, respectively. As shown in Figs. 3(b) and 3(c), $\mathrm{M}^{2}-\mathrm{H}$ and $\left(\partial \mathrm{M}^{2} / \partial \mathrm{H}\right)-\mathrm{H}$ curves can be used to predict the nature of $\lambda$ and piezomagnetic coefficient (q) under the constant stress condition. It is well-known that
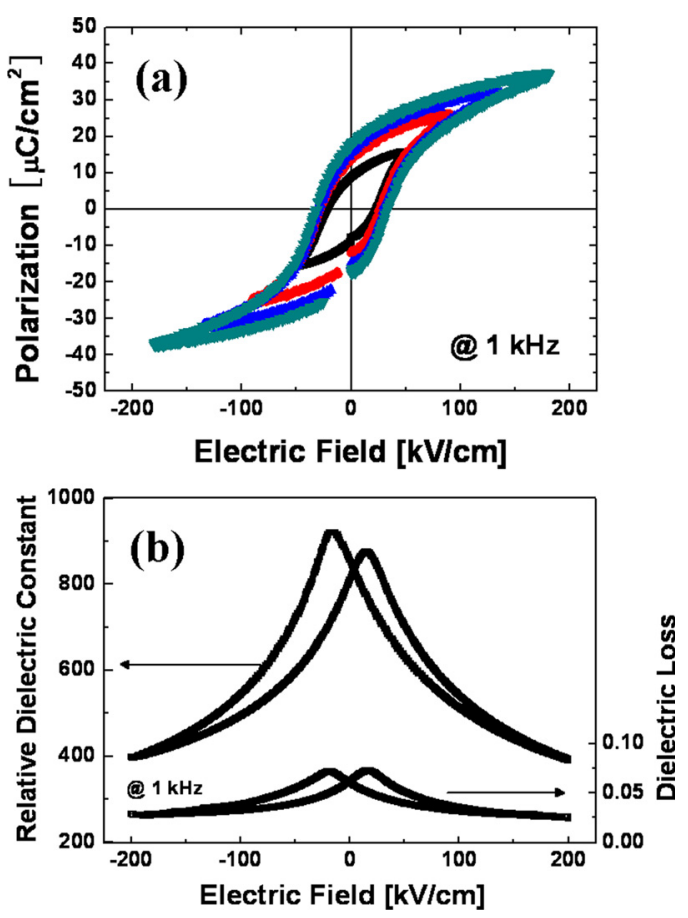

FIG. 2. Electrical properties of the PZT film on Pt/NZF substrate: (a) polarization-electric field curves and (b) dielectric-electric field properties. 

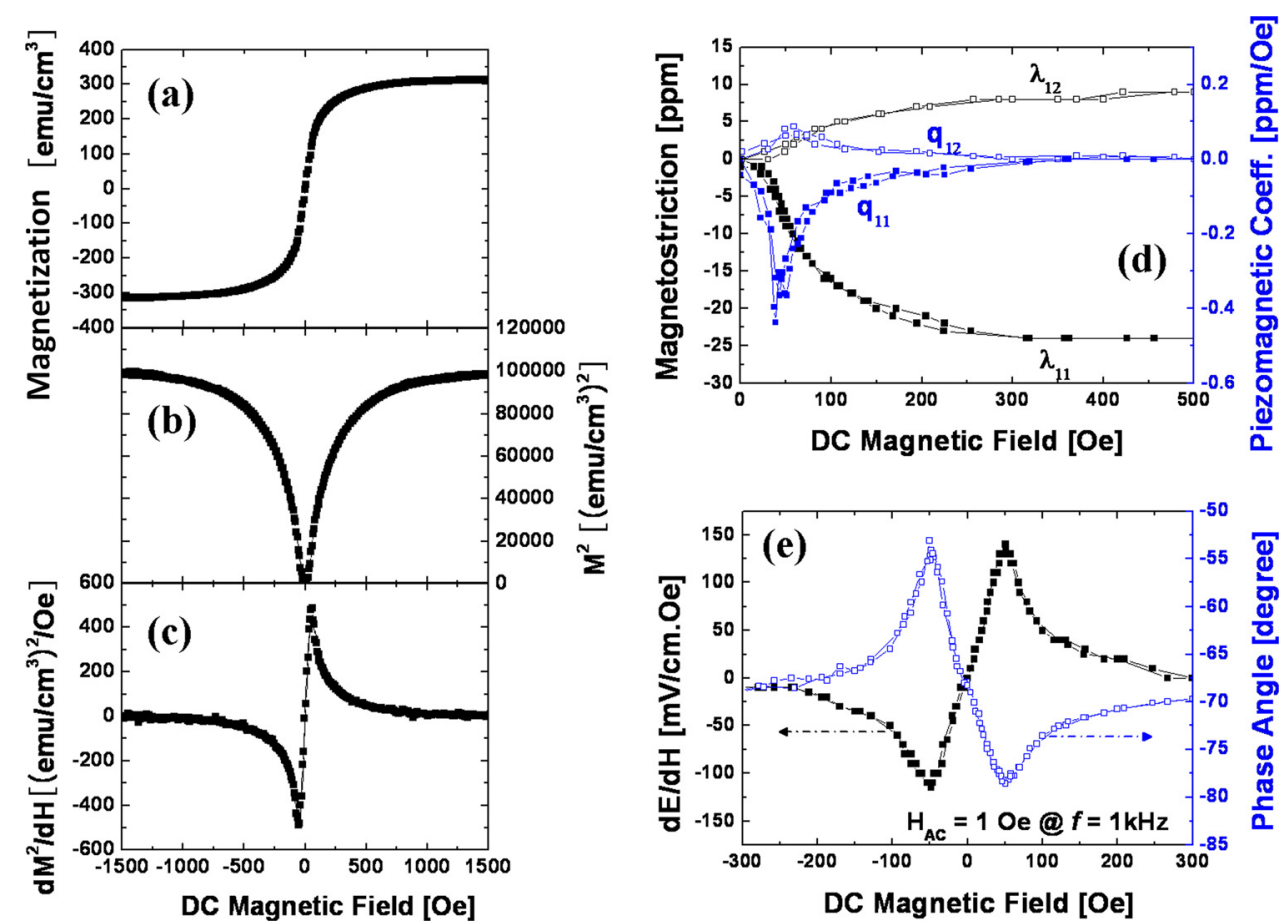

FIG. 3. (a) Magnetization-magnetic field (M-H) response, (b) square magnetization-magnetic field $\left(\mathrm{M}^{2}-\mathrm{H}\right)$, (c) differential of square magnetizationmagnetic field [(dM2/dH)-H], (d) magnetostriction and piezomagnetic coefficient for varying magnitudes of DC magnetic field, and (e) magnetoelectric coefficient and phase angle shift as a function of DC magnetic field under constant AC magnetic field condition of 1 Oe at $f=1 \mathrm{kHz}$. the peak position and magnitude of the ME coefficient for the strain-mediated ME composite are related to the behavior of piezomagnetic coefficients $\left(\mathrm{q}_{\mathrm{ij}}=\mathrm{d} \lambda_{\mathrm{ij}} / \mathrm{dH}\right)$, given by the differential of $\lambda$ with respect to applied magnetic field. ${ }^{17}$

Figure 3(d) shows the variations of $\lambda$ and $\mathrm{q}$ of the PZT/ $\mathrm{Pt} / \mathrm{NZF}$ as a function of $\mathrm{H}_{\mathrm{DC}}$. The coefficients, $\lambda_{11}$ and $\lambda_{12}$, correspond to in-plane magnetostriction coefficient measured parallel and perpendicular to applied $\mathrm{H}_{\mathrm{DC}}$. When $\mathrm{H}_{\mathrm{DC}}$ was applied in longitudinal direction of the specimen, the NZF contracted in the same direction by $24 \mathrm{ppm}$ and elongated in the lateral direction by $9 \mathrm{ppm}$. The parameters $\mathrm{q}_{11}$ and $\mathrm{q}_{12}$ are in-plain piezomagnetic coefficients $\left(\mathrm{q}_{\mathrm{ij}}=\mathrm{d} \lambda_{\mathrm{ij}} / \mathrm{dH}\right)$. The maximum $\mathrm{q}_{11}$ and $\mathrm{q}_{12}$ was obtained at $\mathrm{H}_{\mathrm{DC}}=50$ Oe with values of -0.4 and $0.087 \mathrm{ppm} / \mathrm{Oe}$, respectively. The behaviors of $\lambda$ and $\mathrm{q}$ are identical to that of $\mathrm{M}^{2}$ and $\mathrm{dM}^{2} / \mathrm{dH}$, respectively. Thus, low-field saturated $\mathrm{ME}$ behavior and high $\mathrm{ME}$ coefficient for the PZT film on Pt/NZF substrate can be expected.

For the ME voltage coefficient measurement, the PZT film was poled under DC electric field condition of $200 \mathrm{kV} /$ $\mathrm{cm}$ for $10 \mathrm{~min}$. After aging for $24 \mathrm{~h}, \mathrm{ME}$ output voltage was measured. Figure 3(e) shows the ME coefficient of the PZT film on the substrate as a function of DC magnetic field in longitudinal-transverse (L-T) mode. The peak in ME coefficient of $140 \mathrm{mV} / \mathrm{cm}$ Oe was achieved at low DC bias field of $\mathrm{H}_{\mathrm{DC}}=50 \mathrm{Oe}$ under the constant condition of $\mathrm{H}_{\mathrm{AC}}=1 \mathrm{Oe}$ and $f=1 \mathrm{kHz}$. For comparison, Wang et al. have reported the $\mathrm{ME}$ value of $60 \mathrm{mV} / \mathrm{cm}$ Oe at $\mathrm{H}_{\mathrm{DC}}=\sim 550 \mathrm{Oe}$ by using $300 \mathrm{~nm}$-thick PZT film on CFO. ${ }^{18}$ Our ME coefficient value is $2.3 \times$ higher. Furthermore, magnetic sensitivity increased by $10 \times$ while the peak ME position is $10 \times$ lower. This giant ME coupling at low DC magnetic field condition is attributed to effective elastic coupling between the piezoelectric and magnetostrictive phases. A clear phase angle behavior also represents strong magnetic-electric coupling. ${ }^{19}$

In order to understand the nature of this strong elastic coupling, in-situ dynamic strain was measured. Transversal dynamic motion of the PZT/Pt/NZF under the conditions of $\mathrm{H}_{\mathrm{DC}}=50 \mathrm{Oe}$ and $\mathrm{H}_{\mathrm{AC}}=1 \mathrm{Oe}$ at $f=1 \mathrm{kHz}$ was measured through Pt top electrode by using laser vibrometer. Figure 4(a) shows the applied magnetic field waveform at $f=1 \mathrm{kHz}$. The schematic of measurement set-up is illustrated in Fig. 4(b). The marked points from (i) to (ix) in Fig. 4(a) correspond to the displacement image (i)-(ix) in Fig. 4. The magnitude of transverse dynamic motion can be represented by the $\mathrm{q}_{13}$ value $(0.072 \mathrm{ppm} / \mathrm{Oe})$ of the $\mathrm{PZT} / \mathrm{Pt} / \mathrm{NZF}$ structure. A circulating strain behavior was detected by comparing the images shown in Figs. 4(i)-(ix). To date, a direct measurement of the dynamic coupling motion has not been reported. The results clearly indicate that the piezoelectric strain is not intrinsic property of the homogeneous PZT but rather an extrinsic effect of reorientation of the grain averaged "macroscopic ferroelectric domains." Indeed, due to the polarization coupling between neighboring grains (it is conceptually similar to the exchange coupling in the ferromagnetic polycrystalline films), ${ }^{20}$ there is tendency of ferroelectric domains in these grains to adopt the easy polarization directions that are as close to each other as is permitted by the mis-orientation of the grains. As a result, this coupling produces "grain-averaged macroscopic polar domains" encompassing multiple grains with the average polarization directed along the applied field stress in this case. Using phase field modeling, Jin et al. demonstrated the formation of such macroscopic grain-averaged magnetic domains in polycrystalline ferromagnetics which are conceptually similar systems as polycrystalline ferroelectrics. ${ }^{20}$ If we assume that the misoriented grains pin the boundaries of macroscopic polar domains, the conventional mechanism of polarization provided by the domain wall movement under applied field does not become operational, and the only remaining mode of polarization change is rotation of average polarization. We speculate that this is the rotation that was observed in Fig. 4. Domain rotation is much more effective way to produce the switching-induced strain. Thus, the 

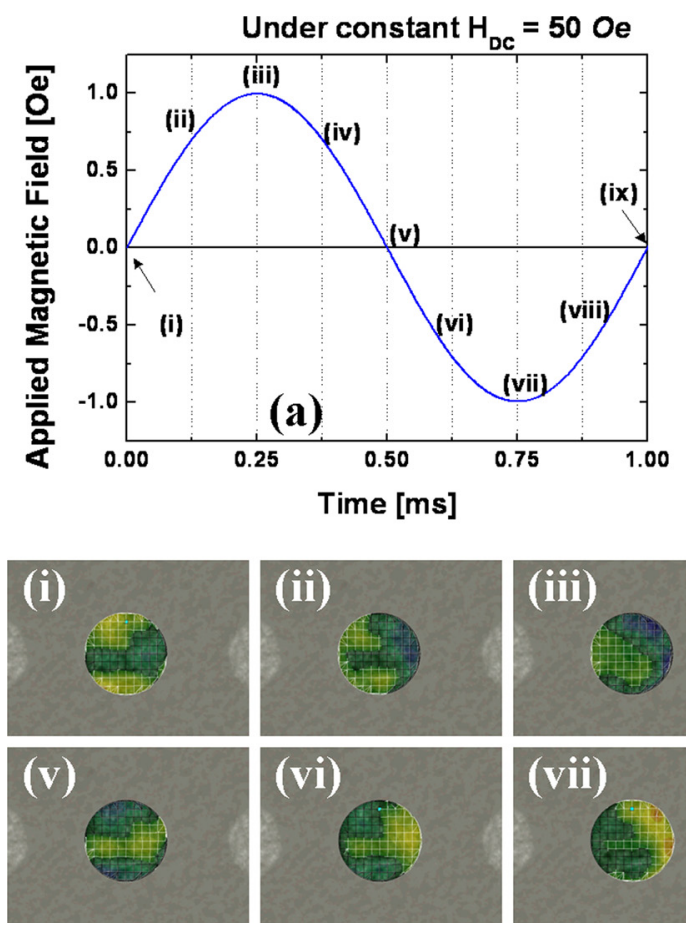
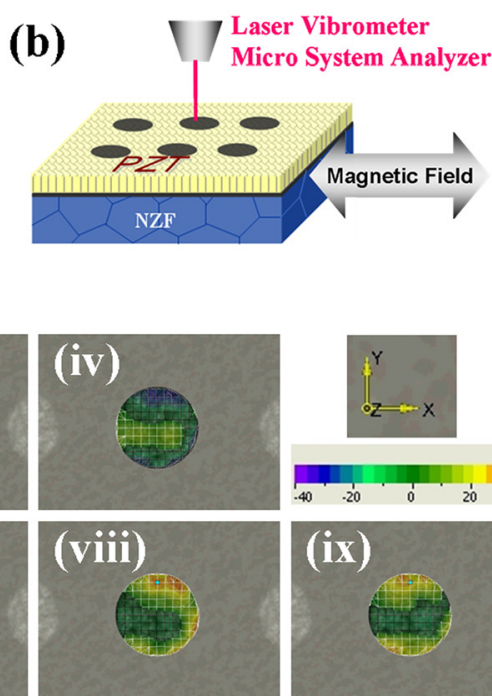
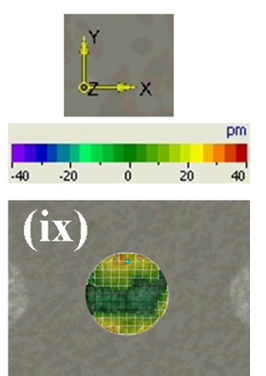

FIG. 4. (a) Applied magnetic field as a function of time under $\mathrm{H}_{\mathrm{AC}}=1 \mathrm{Oe}$ at $f=1 \mathrm{kHz}$ under constant $\mathrm{H}_{\mathrm{DC}}=50 \mathrm{Oe}$, (b) schematic diagram of displacement measurement using a laser analyzer under the magnetic operating condition, and (i)-(ix) dynamic displacement responses corresponding to the input magnetic field. results of this figure indicate that an additional coupling mechanism that may be operating in parallel to the conventional elastic strain induced magnetoelectric coupling which will explain the high response achieved from the composite.

In summary, highly dense $1 \mu \mathrm{m}$-thick PZT film was successfully synthesized on magnetically active platinized NZF substrate. The films showed high ferroelectric and dielectric properties while the substrate provided low field-saturated magnetic properties. The maximum ME response was measured to be $140 \mathrm{mV} / \mathrm{cm}$ Oe at $\mathrm{H}_{\mathrm{DC}}=50$ Oe under the constant condition of $\mathrm{H}_{\mathrm{AC}}=1 \mathrm{Oe}$ and $f=1 \mathrm{kHz}$.

This work was financially supported by Office of Basic Energy Science, Department of Energy, USA (DE-FG0208ER46484).

${ }^{1}$ N. A. Spaldin and M. Fiebig, Science 309, 391 (2005).

${ }^{2}$ J. Ryu, A. Vazquez Carazo, K. Uchino, and H.-E. Kim, J. Electroceram. 7, 17 (2001).

${ }^{3}$ C.-W. Nan, M. I. Bichurin, S. Dong, D. Viehland, and G. Srinivasan, J. Appl. Phys. 103, 301101 (2008).

${ }^{4}$ J. Ma, J. Hu, Z. Li, and C.-W. Nan, Adv. Mater. 23, 1062 (2011).

${ }^{5}$ H. Zheng, J. Wang, S. E. Lofland, Z. Ma, L. Mohaddes-Ardabili, R. Zhao, L. Salamanca-Riba, S. R. Shinde, S. B. Ogale, F. Bai, D. Viehland, Y. Jia, D. G. Schom, M. Wuttig, A. Roytburd, and R. Ramesh, Science 303, 661 (2004).
${ }^{6}$ F. Zavaliche, H. Zheng, L. Mohaddes-Ardabili, S. Y. Yang, Q. Zhan, P. Shafer, E. Reilly, R. Chopdekar, Y. Jia, P. Wright, D. G. Schlom, Y. Suzuki, and R. Ramesh, Nano Lett. 5, 1793 (2005).

${ }^{7}$ J. G. Wan, H. Zhang, X. Wang, D. Pan, J.-M. Liu, and G. Wang, Appl. Phys. Lett. 89, 122914 (2006).

${ }^{8}$ J.-P. Zhou, H. He, Z. Shi, and C.-W. Nan, Appl. Phys. Lett. 88, 013111 (2006).

${ }^{9}$ C. W. Nan, G. Liu, Y. H. Lin, and H. D. Chen, Phys. Rev. Lett. 94, 197203 (2005).

${ }^{10}$ T. Wu, M. A. Zurbuchen, S. Saha, R.-V. Wang, S. K. Streiffer, and J. F. Mitchell, Phys. Rev. B 73, 134416 (2006).

${ }^{11}$ C.-S. Park, J.-W. Lee, G.-T. Park, H.-E. Kim, and J.-J. Choi, J. Mater. Res. 22, 1367 (2007).

${ }^{12}$ C.-S. Park, J.-W. Lee, S.-M. Lee, S.-H. Jun, and H.-E. Kim, J. Electroceram. 25, 20 (2010).

${ }^{13}$ G.-T. Park, J.-J. Choi, C.-S. Park, J.-W. Lee, and H.-E. Kim, Appl. Phys. Lett. 85, 2322 (2004).

${ }^{14}$ C.-S. Park and S. Priya, J. Am. Ceram. Soc. 94, 1087 (2011).

${ }^{15}$ S. Ito, K. Aso, Y. Makino, and S. Uedaira, Appl. Phys. Lett. 37, 665 (1980).

${ }^{16}$ V. K. Vlasko-Vlasov, Y. K. Lin, D. J. Miller, U. Welp, G. W. Crabtree, and V. I. Nikitenko, Phys. Rev. Lett. 84, 2239 (2000).

${ }^{17}$ Y. K. Ferisov, V. M. Petrov, and G. Srinivasan, J. Mater. Res. 22, 2074 (2007).

${ }^{18}$ J. Wang, L. Wang, G. Liu, Z. Shen, Y. Lin, and C. W. Nan, J. Am. Ceram. Soc. 92, 2654 (2009).

${ }^{19}$ S. Dong, J. Zhai, J. Li, and D. Viehland, Appl. Phys. Lett. 88, 082907 (2006).

${ }^{20}$ Y. M. Jin, Yu. U. Wang, A. Kazaryan, Y. Wang, D. E. Laughlin, and A. G. Khachaturyan, J. Appl. Phys. 92, 6172 (2002). 